Aubram, D., Rackwitz, F., Wriggers, P., \& Savidis, S. A.

\title{
An ALE method for penetration into sand utilizing optimization-based mesh motion
}

Journal article | Accepted manuscript (Postprint)

This version is available at https://doi.org/10.14279/depositonce-7263

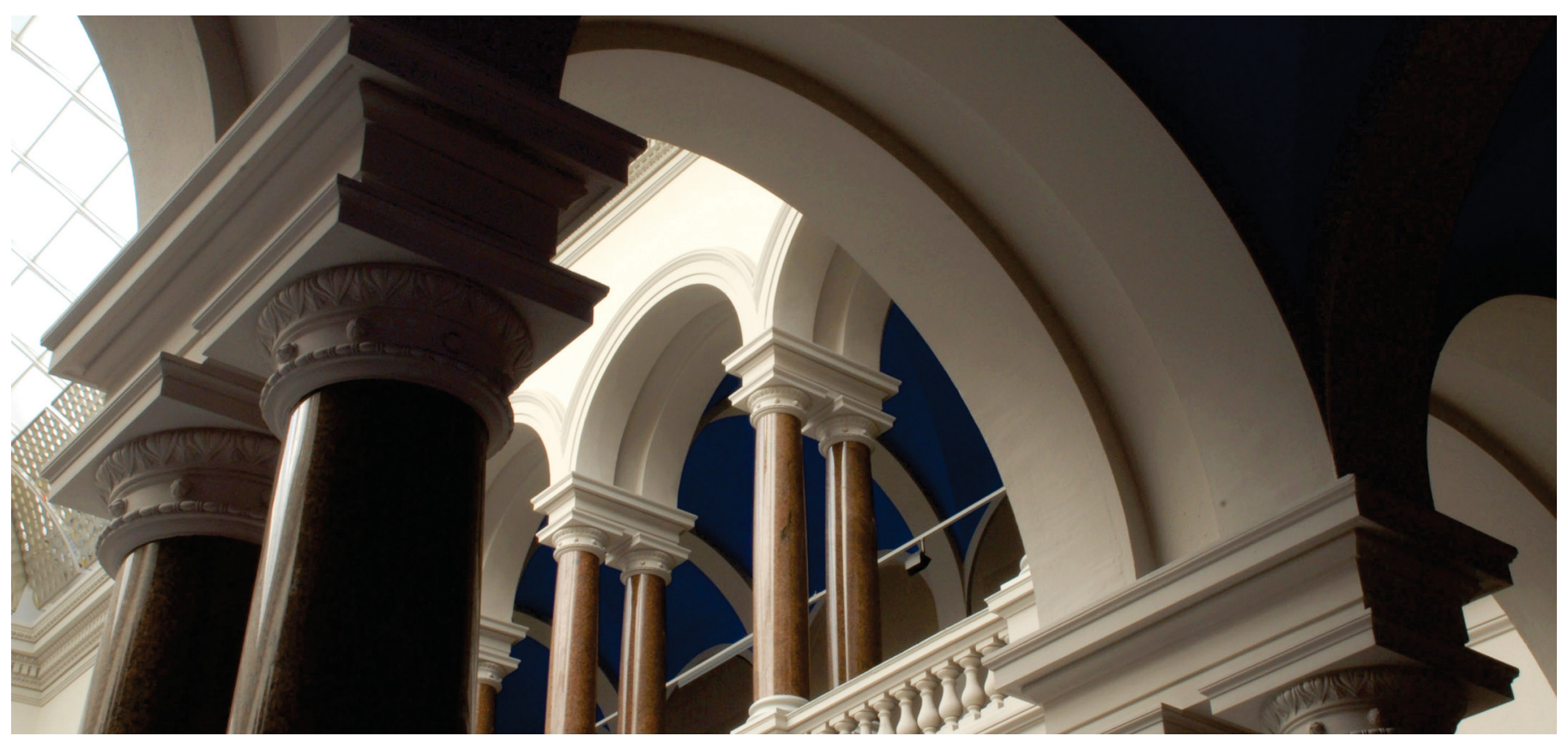

Aubram, D., Rackwitz, F., Wriggers, P., \& Savidis, S. A. (2015). An ALE method for penetration into sand utilizing optimization-based mesh motion. Computers and Geotechnics, 65, 241-249.

https://doi.org/10.1016/j.compgeo.2014.12.012 


\title{
An ALE method for penetration into sand utilizing optimization-based mesh motion
}

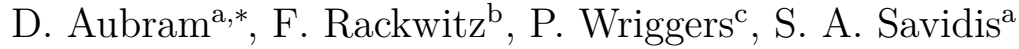 \\ ${ }^{a}$ Chair of Soil Mechanics and Geotechnical Engineering, Technische Universität Berlin, \\ Secr. TIB1-B7, Gustav-Meyer-Allee 25, D-13355 Berlin, Germany \\ ${ }^{b}$ Department of Geotechnical Engineering, Ostbayerische Technische Hochschule \\ Regensburg, Prüfeninger Str. 58, D-93049 Regensburg, Germany \\ ${ }^{c}$ Institute of Continuum Mechanics, Gottfried Wilhelm Leibniz Universität Hannover, \\ Appelstr. 11, D-30167 Hannover, Germany
}

\begin{abstract}
The numerical simulation of penetration into sand is one of the most challenging problems in computational geomechanics. The paper presents an arbitrary Lagrangian-Eulerian (ALE) finite element method for plane and axisymmetric quasi-static penetration into sand which overcomes the problems associated with the classical approaches. An operator-split is applied which breaks up solution of the governing equations over a time step into a Lagrangian step, a mesh motion step, and a transport step. A unique feature of the ALE method is an advanced hypoplastic rate constitutive equation to realistically predict stress and density changes within the material even at large deformations. In addition, an efficient optimization-based algorithm has been implemented to smooth out the non-convexly distorted mesh regions that occur below a penetrator. Applications to shallow penetration and pile penetration are given which make use of the developments.
\end{abstract}

Keywords: arbitrary Lagrangian-Eulerian, large deformations, penetration, sand, pile

${ }^{*}$ Corresponding author. Tel.: +49 (0)30 31472349; Fax: +49 (0)30 31472343. E-mail address: daniel.aubram@tu-berlin.de (D. Aubram)

NOTICE: This is the author's version of a work that was accepted for publication in Computers and Geotechnics. A definitive version of this article is published in: D. Aubram, F. Rackwitz, P. Wriggers, S.A. Savidis: An ALE method for penetration into sand utilizing optimization-based mesh motion. Computers and Geotechnics 65: 241-249, (2015). doi:10.1016/j.compgeo.2014.12.012 


\section{Introduction}

Penetration into sand is one of the oldest problems in geomechanics, and it turned out to be also one of the most challenging. Rigorous modeling is very difficult because of the large local deformations in the vicinity of the penetrator, the evolution of material interfaces and free surfaces, the changing contact conditions, the large stiffness variations, and the complex nonlinear behavior of the granular material. Therefore, penetration into sand has not been extensively explored so far from a numerical viewpoint.

The finite element method (FEM) is the dominating tool in computational geomechanics because of its broad applicability and technological sophistication. Typical models are based on a Lagrangian description, which is in many respects the most attractive approach for problems where pathdependent material response and evolving interfaces are present. However, if material deformations are large severe element distortion may occur which may slow down or even terminate the calculation. Element distortion will not occur in the Eulerian methods commonly used in computational fluid dynamics (CFD) because the mesh is kept spatially fixed. However, tracking

of free surfaces and material motion becomes non-trivial because the material is allowed to flow through the mesh.

The arbitrary Lagrangian-Eulerian (ALE) methods have been developed in order to overcome the difficulties arising from the purely Lagrangian and Eulerian approaches, and to combine their advantages $[1,2,3,4,5,6]$. The major advantage of ALE is that the computational mesh is regarded as a independent reference domain. As a consequence, the ALE mesh can be continuously smoothed so that element quality remains acceptable during the entire calculation. Mesh connectivity is kept unchanged, hence the solution variables can be remapped onto the improved mesh by using conservative CFD advection algorithms.

Despite their popularity in fluid-structure interaction and solid mechanics, applications of ALE methods to soil mechanical problems are still rare. Examples include $[7,8,9,10,11,12]$, but the employed methods reveal two limitations when applied to penetration into sand. First, common algorithms for node relocation are not qualified to smooth the non-convex mesh regions that inevitably occur during penetration of blunt bodies. As a consequence, the range of problems that can be addressed by these ALE methods is not much larger than for a purely Lagrangian approach. Second, the constitutive equations involved (Mohr-Coulomb, Drucker-Prager, modi- 
fied Cam-Clay, etc.) are not able to realistically reproduce the stress- and density-dependent response of sand under monotonic and cyclic loading.

The mechanical behavior of sand is very complex and has several influencing factors. Amongst others, it is generally a function of the effective stress state, the relative density, and the material history due to monotonic or cyclic loading [13]. An important characteristic that distinguishes the behavior of sand from that of common solids is dilatancy, which has been shown to depend on both the effective stress state and density state [14]. Moreover, sand shows asymptotic behavior [15] and reaches a critical state after monotonic loading paths starting from a particular stress and density state.

The best models currently available to properly reproduce this complex nonlinear behavior are phenomenological constitutive equations that rely on the continuum representation of sand. However, there are just a few constitutive equations for granular solids available which need only a single set of material constants and are then able to simulate the mechanical behavior at finite deformations and under complex loading paths over the wide range of densities and stress states present during penetration processes.

In this paper we present an ALE method which is particularly suitable for penetration into sand. Its unique features are (i) an advanced constitutive equation for sand and (ii) an efficient and robust optimization-based mesh regularization algorithm which delivers excellent results even on nonconvexly distorted domains. For simplicity, we consider sand as a single-phase medium, being either dry or fully saturated and locally drained. Moreover, only plane strain and axisymmetric quasi-static problems are considered in the numerical treatment. The penetrator is assumed to be either smooth (zero friction) or perfectly rough (no sliding). The 3-node triangle element $[16,17]$ is used for the spatial discretization despite of its tendency to lock up. However, the impact of this undesirable feature is not much significant provided that special mesh pattern are used.

The remainder of this paper is structured as follows. Section 2 presents the governing equations associated with our ALE approach. The steps of the operator-split solution procedure are described in detail in Section 3, and Section 4 shows example applications concerning shallow penetration and pile penetration into sand. The paper closes with some concluding remarks in Section 5. 


\section{Governing equations}

The following section introduces basic equations of ALE continuum mechanics. The full derivation of these equations is beyond the scope of the paper and can be found in [18].

\subsection{Kinematics and balance principles}

Let $\mathcal{B} \subset \mathcal{S}$ be the reference configuration of a material body and $\varphi_{t}: \mathcal{B} \rightarrow$ $\mathcal{S}$ its motion in the ambient space $\mathcal{S}$, with time $t \in\left[t_{0}, T\right]$ and $\varphi_{t}(\cdot)=\varphi(\cdot, t)$ at fixed $t$. An arbitrary subset $\mathcal{R} \subset \mathcal{S}$ is referred to as a reference domain provided that there exist a diffeomorphism $\Psi_{t}: \mathcal{R} \rightarrow \mathcal{B}$ and an embedding $\Phi_{t}: \mathcal{R} \rightarrow \mathcal{S}$ such that

$$
\varphi_{t}=\Phi_{t} \circ \Psi_{t}^{-1}
$$

where $\circ$ indicates composition of maps. The material and mesh velocities are $\boldsymbol{v}_{t} \stackrel{\text { def }}{=}\left(\frac{\partial}{\partial t} \varphi_{t}\right) \circ \varphi_{t}^{-1}$ and $\boldsymbol{w}_{t} \stackrel{\text { def }}{=}\left(\frac{\partial}{\partial t} \Phi_{t}\right) \circ \Phi_{t}^{-1}$, respectively, and their difference

$$
\boldsymbol{c} \stackrel{\text { def }}{=} \boldsymbol{v}-\boldsymbol{w}
$$

is called the convective velocity. Since our ALE method enforces the zero-flux constraint $\boldsymbol{c} \cdot \boldsymbol{n}=0$ on $\partial \Phi_{t}(\mathcal{R})=\partial \varphi_{t}(\mathcal{B})$, where $\boldsymbol{n}$ is the field of outward normals, it falls into the category of single-material or simplified ALE methods [19].

Let $q$ be the spatial description of the physical field under consideration and $\hat{q} \stackrel{\text { def }}{=} q \circ \Phi$ its referential or ALE description. The general ALE conservation form of a balance principle then reads

$$
\frac{\partial \hat{q} J_{\Phi}}{\partial t}+J_{\Phi}(\operatorname{div}(q \otimes \boldsymbol{c})) \circ \Phi=J_{\Phi}(S \circ \Phi)
$$

where div is the divergence operator on the ambient space, $J_{\Phi}$ is the Jacobian of $\Phi$, and $S$ is a source term. Our ALE method is based on the common operator-split solution strategy [19], which divides (3) into

$$
\dot{q}=S-q \operatorname{div} \boldsymbol{v} \quad \text { and } \quad \frac{\partial \hat{q} J_{\Phi}}{\partial t}+J_{\Phi} \operatorname{div}(q \otimes \boldsymbol{c}) \circ \Phi=0
$$

where $\dot{q}$ denotes the material time derivative of $q$.

The first equation in (4) accounts for the sources by ignoring convective transport $(\boldsymbol{c}=\mathbf{0})$. As the motion of the reference domain coincides with the 
material motion, the description of motion is (updated) Lagrangian. The second equation formalizes the transport resp. advection of $q$ on the reference domain moving with convective velocity $\boldsymbol{c} \neq \mathbf{0}$ and without any external source or material evolution.

In the present research, the balance principles governing quasi-static penetration into sand are conservation of mass and balance of momentum. The updated Lagrangian form $(4)_{1}$ of these balance principles reads

$$
\dot{\rho}=-\rho \operatorname{div} \boldsymbol{v} \quad \text { and } \quad \rho \boldsymbol{a}=\rho \boldsymbol{b}+\operatorname{div} \boldsymbol{\sigma},
$$

respectively, in which $\rho$ is the mass density, $\boldsymbol{\sigma}=\boldsymbol{\sigma}^{\mathrm{T}}$ is the symmetric Cauchy stress field, $\boldsymbol{b}$ is an applied force per unit mass, and $\boldsymbol{a}$ is a prescribed static acceleration field.

We note that, by assuming $\varphi\left(\mathcal{B}, t_{0}\right)=\mathcal{B}$, conservation of mass is equivalent to

$$
\frac{\partial J}{\partial t}=J(\operatorname{tr} \boldsymbol{d}) \circ \varphi \quad \text { in conjunction with } \quad \rho_{0}=(\rho \circ \varphi) J,
$$

where $J$ is the Jacobian of $\varphi, \boldsymbol{d}=\frac{1}{2}\left(\boldsymbol{\nabla} \boldsymbol{v}+(\boldsymbol{\nabla} \boldsymbol{v})^{\mathrm{T}}\right)$ is the spatial rate of deformation, tr returns the trace of a second-order tensor, and $\rho_{0}$ is the mass density at $t=t_{0}$.

\subsection{Constitutive equations}

Spatial rate constitutive equations based on the corotational objective Zaremba-Jaumann or Green-Naghdi stress rates are commonly used in solid mechanical ALE applications [19]. The present method considers material response described by a constitutive equation taking the general form

$$
\stackrel{\nabla}{\stackrel{\text { def }}{=}} \boldsymbol{h}(\boldsymbol{\sigma}, \boldsymbol{\alpha}, \boldsymbol{d}) \stackrel{\text { def }}{=} \boldsymbol{m}(\boldsymbol{\sigma}, \boldsymbol{\alpha}, \boldsymbol{d}): \boldsymbol{d}
$$

where $\stackrel{\nabla}{\boldsymbol{\sigma}}=\dot{\boldsymbol{\sigma}}-\boldsymbol{\omega} \cdot \boldsymbol{\sigma}+\boldsymbol{\sigma} \cdot \boldsymbol{\omega}$ is the Zaremba-Jaumann rate of Cauchy stress, $\boldsymbol{\omega}=\frac{1}{2}\left(\boldsymbol{\nabla} \boldsymbol{v}-(\boldsymbol{\nabla} \boldsymbol{v})^{\mathrm{T}}\right)$ is the vorticity of motion, $\boldsymbol{\alpha}=\left\{\alpha_{1}, \ldots, \alpha_{N}\right\} \stackrel{\text { def }}{=}\left\{\alpha_{k}\right\}$ is a set of internal material state variables in addition to stress, and $\boldsymbol{m}$ is the fourth-order material tangent tensor. Elements of $\boldsymbol{\alpha}$ are assumed to have evolution equations comparable to $(7)$, i.e. $\stackrel{\nu_{\alpha}}{{ }_{k}} \stackrel{\text { def }}{=} j_{k}(\boldsymbol{\sigma}, \boldsymbol{\alpha}, \boldsymbol{d})$.

During penetration, sand or granular material in general is subjected to complex loading paths and will undergo changes in relative density and effective stress state over a wide range, depending on its initial state, and its dila- 
tancy and asymptotic response. The description of this mechanical behavior is very complicated and cannot be reproduced by the classical plasticity models, including the Mohr-Coulomb, Drucker-Prager, or Cam-Clay models [13]. In order to reach the objective of realistic numerical simulation of penetration into sand, the ALE method is combined with an advanced rate constitutive equation based on the hypoplasticity framework [20]. Hypoplasticity does not distinguish between elastic and plastic strains. It can be understood as a generalization of hypoelasticity [21] insofar as the response function $\boldsymbol{h}$ resp. the material tangent $\boldsymbol{m}$ introduced by (7) is generally nonlinear in $\boldsymbol{d}$ in order to describe dissipative behavior.

The particular hypoplastic model for sand used here has been proposed in $[22,23]$ and includes the extensions of $[24,25]$ to account for limit states and the mechanical behavior under cyclic loading. For completeness, the full mathematical expressions of the constitutive functions are attached in Appendix A. The hypoplastic model has several advantages over classical plasticity models commonly used in soil mechanical ALE methods in the literature. The main advantage is the use of the void ratio $e$ and of the socalled intergranular strain $\boldsymbol{\delta}$ capturing loading history in the set of material state variables. As a result, the behavior of sand for various monotonic and cyclic loading programs can be described by using only a single set of hypoplastic material constants $[24,25,26]$. There might be indeed alternative models possessing comparable features, but to the knowledge of the authors these have not been used in ALE calculations yet.

\section{Numerical implementation}

\subsection{Operator-split solution procedure}

The operator-split (4) applied to the governing equations discussed in the previous section results in the two sets of equations (compositions with point mappings are dropped)

$$
\begin{aligned}
\text { balance of momentum } & \rho \boldsymbol{a}=\rho \boldsymbol{b}+\operatorname{div} \boldsymbol{\sigma}, \\
\text { conservation of mass } & \frac{\partial J}{\partial t}=J \operatorname{tr} \boldsymbol{d} \text { i.c.w. } \rho_{0}=\rho J, \\
\text { rate constitutive equation } & \stackrel{\nabla}{\boldsymbol{\sigma}}=\boldsymbol{h}(\boldsymbol{\sigma}, \boldsymbol{\alpha}, \boldsymbol{d}), \\
\text { evolution equation } & \stackrel{\nabla}{\alpha}_{k}=j_{k}(\boldsymbol{\sigma}, \boldsymbol{\alpha}, \boldsymbol{d}),
\end{aligned}
$$


and

$$
\begin{aligned}
\text { transport of Jacobian of } \varphi & \frac{\partial \hat{J} J_{\Phi}}{\partial t}+J_{\Phi} \operatorname{div}(J \boldsymbol{c})=0, \\
\text { transport of stress } & \frac{\partial \hat{\boldsymbol{\sigma}} J_{\Phi}}{\partial t}+J_{\Phi} \operatorname{div}(\boldsymbol{\sigma} \otimes \boldsymbol{c})=\mathbf{0}, \\
\text { transport of state variables } & \frac{\partial \hat{\alpha}_{k} J_{\Phi}}{\partial t}+J_{\Phi} \operatorname{div}\left(\alpha_{k} \otimes \boldsymbol{c}\right)=\mathbf{0},
\end{aligned}
$$

ALE finite element methods approximate the reference domain $\mathcal{R}$ by a disjoint union of finite elements. The time interval of interest is approximated by a sequence $\left(t_{0}, \ldots, t_{n+1}=t_{n}+\Delta t, \ldots, T\right)$ of discrete time steps. The solution in time is carried out in calculational cycles consisting of three steps: a Lagrangian step, a mesh motion step, and a transport step. Conceptually, the time associated with the end of the Lagrangian step is denoted by $t_{n+1}^{-}$, whereas the time associated with the end of both the mesh motion step and the transport step is $t_{n+1}^{+}$. However, physical time elapses only during in the Lagrangian step.

Alg. 1 provides the pseudocode of the proposed incremental operatorsplit ALE finite element solution procedure for quasi-static problems. The essential steps are described in the following sections.

\subsection{Lagrangian step}

Standard nonlinear finite element techniques are employed to solve the initial boundary value problem in the updated Lagrangian description and to advance balance of momentum (8) implicitly in time. The Newmark- $\beta$ method in conjunction with Newton's method is applied for this purpose. Without contact constraints the resulting system of linearized equations, in matrix notation, is

$$
\boldsymbol{K}_{n+1}^{i} d \boldsymbol{u}_{n+1}^{i}=\boldsymbol{b}_{n+1}^{i}, \quad \text { with } \quad \boldsymbol{b}_{n+1}^{i}=\boldsymbol{f}_{n+1}^{\text {ext }}-\boldsymbol{M} \boldsymbol{a}-\boldsymbol{f}^{\mathrm{int}}\left(\boldsymbol{u}_{n+1}^{i}\right) .
$$

$\boldsymbol{M}$ is the global mass matrix of the finite element assembly, $\boldsymbol{f}^{\text {int }}$ and $\boldsymbol{f}^{\text {ext }}$ are the global internal and external nodal force vectors, respectively, and $\boldsymbol{K}_{n+1}^{i}$ is the global stiffness matrix referring to the $i$-th iteration of the solution at time $t_{n+1}^{-}$. The external nodal acceleration vector $\boldsymbol{a}$ is required to simulate gravity in quasi-static analysis. Detailed derivations of (15) can be found, for example, in $[16,17]$ and will not be repeated here. 


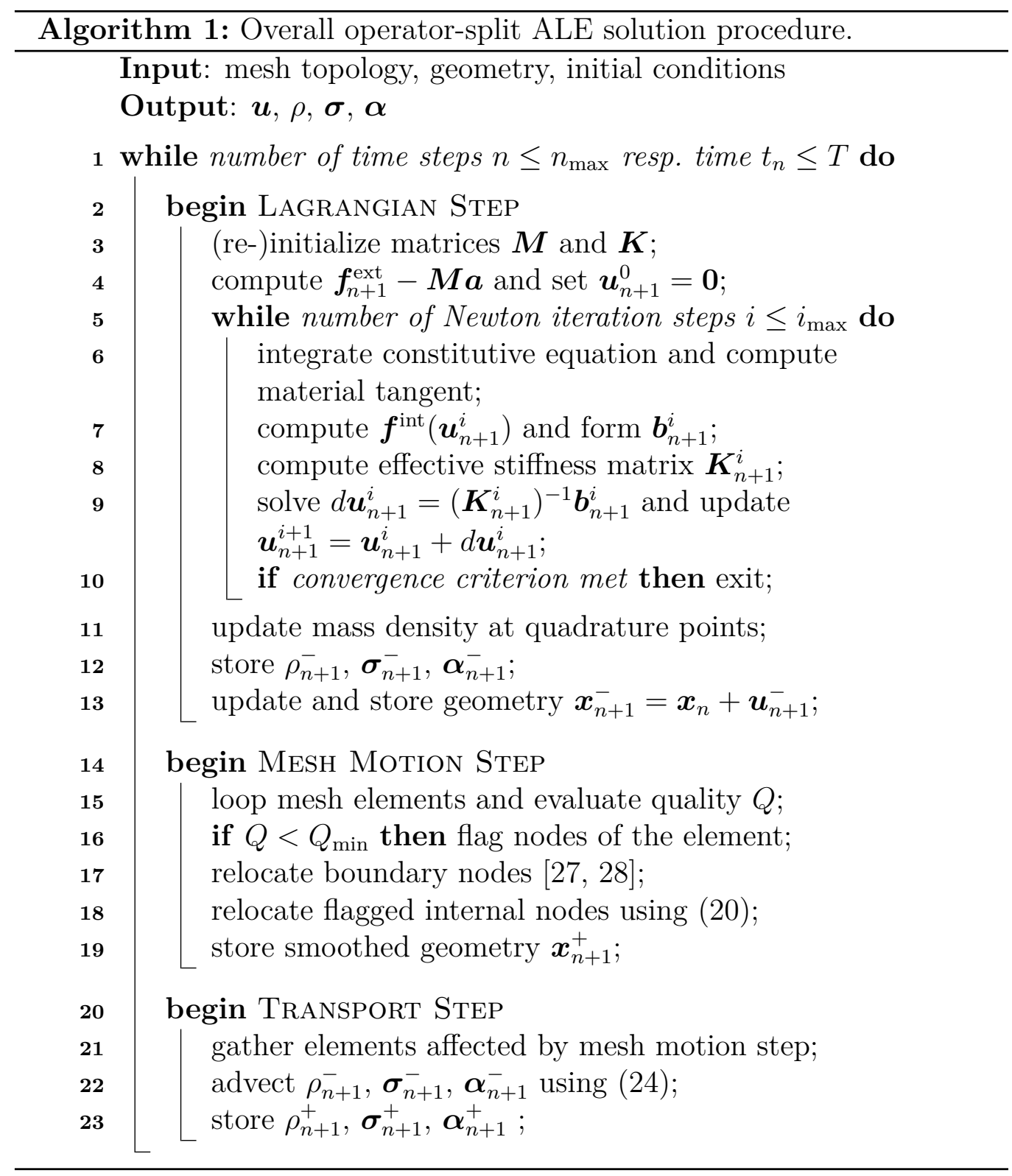


The current accumulated incremental displacements in the $i$-th iteration during the Lagrangian step, $\boldsymbol{u}_{n+1}^{i}=\sum_{k=0}^{i} d \boldsymbol{u}_{n+1}^{k}$, are passed to the procedures which integrate the rate equations (9)-(11) in time at the quadrature points of the finite elements; note that $\boldsymbol{u}_{n}=\mathbf{0}$ since no displacements are stored. A time-centered approximation is used in accordance with [29], leading to an algorithmic finite strain increment computed as

$$
\Delta \boldsymbol{\varepsilon}_{n+1 / 2} \stackrel{\text { def }}{=} \frac{1}{2}\left(\boldsymbol{\nabla}_{n+1 / 2} \boldsymbol{u}+\left(\boldsymbol{\nabla}_{n+1 / 2} \boldsymbol{u}\right)^{\mathrm{T}}\right)
$$

where $\boldsymbol{\nabla}_{n+1 / 2} \boldsymbol{u}=2\left(\boldsymbol{f}_{n+1}-\boldsymbol{I}\right)\left(\boldsymbol{f}_{n+1}+\boldsymbol{I}\right)^{-1}$ and $\boldsymbol{f}_{n+1}$ is the relative incremental deformation gradient of the configuration at $t=t_{n+1}$, represented by the nodal positions $\boldsymbol{x}_{n+1}^{i}=\boldsymbol{x}_{n}+\boldsymbol{u}_{n+1}^{i}$, with respect to the configuration $\boldsymbol{x}_{n}$.

Since mass is not automatically conserved in ALE methods, a solution is required to the initial value problem (9) subject to $\left.J\right|_{t_{n}}=J_{n}$. Into our ALE method we implemented an approximation to the solution at time $t_{n+1}$ using the midpoint rule [18]:

$$
J_{n+1}=J_{n}\left(1-\frac{1}{2} \operatorname{tr} \Delta \varepsilon_{n+1 / 2}\right)^{-1}\left(1+\frac{1}{2} \operatorname{tr} \Delta \varepsilon_{n+1 / 2}\right), \quad \text { so } \quad \rho_{n+1}=\frac{\rho_{0}}{J_{n+1}} .
$$

To meet the requirement of material frame indifference [21] on a discrete level, the incrementally objective algorithm of [30] has been implemented for the integration of (10) and (11). According to this algorithm, integration is carried out with respect to a corotated configuration of the material body. Concerning the implementation of the hypoplastic model used here, the reader is referred to [18] for details.

\subsection{Mesh motion step}

To improve mesh quality, a node located at $\boldsymbol{x}_{n+1}^{-}=\boldsymbol{x}_{n}+\boldsymbol{u}_{n+1}$ after the Lagrangian step will be moved to $\boldsymbol{x}_{n+1}^{+}$without changing mesh connectivity by making use of an appropriate mesh smoothing algorithm. The majority of ALE methods on unstructured meshes employs smoothing algorithms which are not governed by quality evolution, including heuristic procedures $[27,12,31]$ and physically-based smoothing $[32,9]$. Even though these methods are computationally attractive, they cannot ensure that any mesh processed is not worse than before. This is particularly true if the mesh is not convex or becomes non-convexly distorted, as during the penetration into sand investigated here. 
Non-convex meshes are efficiently smoothed by optimization-based algorithms [33, 34, 35, 36]. The particular algorithm implemented into the ALE method is described in detail in [28]. Hence, only a few aspects will be addressed here.

The proposed algorithm assumes that all nodes of the mesh are allowed to be moved, except for the boundary nodes that essentially define the shape of the meshed domain (corner nodes). Smoothing is initiated if at least one mesh element fails a check of geometric quality, and then the nodes of these elements are flagged. The set of flagged nodes is then processed by local smoothing algorithms for boundary nodes and internal nodes. The globally improved mesh is obtained by looping over the flagged nodes repeatedly in an iterative fashion.

The heuristic averaging algorithm of Aymone et al. [27] efficiently smoothes mesh boundaries on a local level. Compared to the boundary mesh, smoothing of the internal mesh is much more complicated and can be stated as an optimization problem [37]:

$$
\text { minimize } W(\boldsymbol{x}) \quad \text { subject to } \boldsymbol{x} \in \mathbb{R}^{m} \text {. }
$$

The function $W$ is called the objective function and probably has a minimizer $\boldsymbol{x}^{\prime} \in \arg \min _{\boldsymbol{x} \in \mathbb{R}^{m}} W(\boldsymbol{x})$. Assuming the objective function to be twice continuously differentiable, then its gradient $\boldsymbol{\nabla} W(\boldsymbol{x}) \in \mathbb{R}^{m}$ and Hessian $\boldsymbol{H}_{W}(\boldsymbol{x}) \in \mathbb{R}^{m \times m}$ at point $\boldsymbol{x} \in \mathbb{R}^{m}$ will exist.

The choice of an objective function is crucial to the success of mesh smoothing. The function considered here takes the form [35]

$$
W(\boldsymbol{x}) \stackrel{\text { def }}{=} \sum_{n_{\mathrm{el}}} w(\boldsymbol{x}), \quad \text { where } \quad w(\boldsymbol{x}) \stackrel{\text { def }}{=} \frac{R(\boldsymbol{x})}{R_{\mathrm{ref}}}\left(\frac{R(\boldsymbol{x})}{r(\boldsymbol{x})}\right)^{3},
$$

$r$ and $R$ are the incircle and circumcircle radius of a triangle element, respectively, $R_{\text {ref }}=1.0$ is a reference radius, and $n_{\mathrm{el}}$ is the number of elements in the ball $\mathcal{B}(\boldsymbol{x})=\bigcup_{n_{\mathrm{el}}} \Omega(\boldsymbol{x})$ sharing the internal node $\boldsymbol{x}$.

For a given $\boldsymbol{x}^{j}$, with $j \in \mathbb{N}$, the iterative procedure to find the minimizer takes the form

$$
\boldsymbol{x}^{j+1}=\boldsymbol{x}^{j}+\lambda^{j} \boldsymbol{d}^{j} \quad \text { with } \quad \lim _{j \rightarrow \infty} \boldsymbol{x}^{j+1}=\boldsymbol{x}^{\prime},
$$

where $\boldsymbol{d} \in \mathbb{R}^{m}$ is a descent direction of $W$ at $\boldsymbol{x}$ satisfying $(\boldsymbol{\nabla} W(\boldsymbol{x}))^{\mathrm{T}} \boldsymbol{d}<0$. 
Once a starting point $\boldsymbol{x}^{j=0}$, a step size $\lambda^{j=0}>0$, and a tolerance $\varepsilon>0$ have been specified, a termination criterion of the form

$$
\left\|\nabla W\left(\boldsymbol{x}^{j}\right)\right\|<\varepsilon
$$

is checked. If this criterion is met, then $\boldsymbol{x}^{j} \approx \boldsymbol{x}^{\prime}$ is an approximation to the minimizer of $W$. If the criterion is not met, Newton's direction $\boldsymbol{d}^{j}=-\left(\boldsymbol{H}_{W}^{-1} \boldsymbol{\nabla} W\right)\left(\boldsymbol{x}^{j}\right)$ is computed provided that the Hessian is regular and positive definite, otherwise the steepest descent $-\boldsymbol{\nabla} W\left(\boldsymbol{x}^{j}\right)$ will be used. After the descent direction has been computed, a so-called line search is carried out in order to determine the step size $\lambda^{j}$ satisfying $W\left(\boldsymbol{x}^{j}+\lambda^{j} \boldsymbol{d}^{j}\right)<W\left(\boldsymbol{x}^{j}\right)$.

\subsection{Transport step}

In the transport step, the solution variables obtained after the Lagrangian step at time $t=t^{-}$are remapped onto the updated mesh at time $t=t^{+}$; the subscript $n+1$ is dropped for notational brevity. The associated set of equations (12)-(14) can be equivalently expressed in form of the generic ALE conservation law $(4)_{2}$, with $q \in\{J, \boldsymbol{\sigma}, \boldsymbol{\alpha}\}$ and $\boldsymbol{c}=\boldsymbol{v}-\boldsymbol{w}=\left(\boldsymbol{x}^{-}-\boldsymbol{x}^{+}\right) / \Delta t$, where $\Delta t \stackrel{\text { def }}{=} t^{+}-t^{-}$is a pseudo time increment. The solution state is frozen, so that time-dependency of the transported variable is implied by the moving mesh only.

The current transport step applies a finite volume strategy. Finite volume methods have traditionally been pursued by the CFD community $[38,39]$ and have earned broad recognition in the history of development of ALE and related methods $[2,5,6,40,12,41]$. They are conservative because they solve the integral form of the conservation law $(4)_{2}$,

$$
\frac{\mathrm{d}}{\mathrm{d} t} \int_{\mathcal{V}_{j}} q \mathrm{~d} v+\int_{\partial \mathcal{V}_{j}} q \boldsymbol{c} \cdot \boldsymbol{n} \mathrm{d} a=0, \quad \text { subject to }\left.q\right|_{t=t^{-}}=q^{-}
$$

The first term accounts for the time-dependency of the control volume $\mathcal{V}_{j} \stackrel{\text { def }}{=} \Phi_{t}\left(\mathcal{W}_{j}\right)$, where $\mathcal{W}_{j} \subset \mathcal{R}$ and $t \in\left[t^{-}, t^{+}\right]$. The second term in $(22)$ expresses the convective flux of the variable $q$ across a control volume boundary. The current method approximates this term by a weighted donor-cell (Godunov-type) flux function. Donor-cell advection is used in many ALE codes $[2,6,40,12,41]$ because it has some highly desirable features [38, 39].

Equation (22) is discretized in space by using a control volume tessellation, which currently coincides with the finite element mesh since all variables 
are stored at the single quadrature point in each linear triangle. Discretization in time is done by the forward Euler method. Moreover, in order to solve the plane and axisymmetric problems considered in this research, we introduced a pseudo-radius $R$ [42] defined for all $\boldsymbol{x} \stackrel{\text { def }}{=}\left(z^{1}, z^{2}\right)^{\mathrm{T}} \in \mathbb{R}^{2}$ through

$$
R(\boldsymbol{x}) \stackrel{\text { def }}{=} 1-\beta+\beta z^{1}(\boldsymbol{x}), \quad \text { where } \beta \in\{0,1\} .
$$

The plane case, $R=1$, is recovered if $\beta=0$, whereas $\beta=1$ is associated with the axisymmetric case $R=z^{1}$, with $z^{1}$ corresponding to the radial coordinate. The resulting transport algorithm then reads [18]

$$
q_{j}^{+}=q_{j}^{-}-\frac{\Delta t}{2 R_{j}^{+} A_{j}^{+}} \sum_{\text {edges }} R_{j, k}^{-} L_{j, k}^{-} F_{j, k}^{V-}\left(q_{k}^{-}-q_{j}^{-}\right)\left(1-\theta \operatorname{sgn}\left(F_{j, k}^{V-}\right)\right),
$$

in which $\theta \in[0,1]$ is the donor cell weighting factor $[2,40]$ and

$$
\begin{aligned}
A_{j} & =\frac{1}{2} \sum_{I=0}^{2}\left(z_{I}^{1} z_{I+1}^{2}-z_{I+1}^{1} z_{I}^{2}\right) \\
R_{j} & =1-\beta+\frac{\beta}{6 A_{j}} \sum_{I=0}^{2}\left(z_{I}^{1}+z_{I+1}^{1}\right)\left(z_{I}^{1} z_{I+1}^{2}-z_{I+1}^{1} z_{I}^{2}\right) \\
L_{j, k} & =\sqrt{\left(z_{I+1}^{1}-z_{I}^{1}\right)^{2}+\left(z_{I+1}^{2}-z_{I}^{2}\right)^{2}} \\
R_{j, k} & =1-\beta+\frac{\beta}{2}\left(z_{I}^{1}+z_{I+1}^{1}\right) \\
F_{j, k}^{V} & =\frac{1}{2}\left(\begin{array}{c}
c_{I}^{1}+c_{I+1}^{1} \\
c_{I}^{2}+c_{I+1}^{2}
\end{array}\right)\left(n_{1}, n_{2}\right)_{I, I+1} \\
& =\left\|\begin{array}{c}
z_{I+1}^{2}-z_{I}^{2} \\
z_{I}^{1}-z_{I+1}^{1}
\end{array}\right\|^{-1}\left(\begin{array}{c}
z_{I+1}^{2}-z_{I}^{2} \\
z_{I}^{1}-z_{I+1}^{1}
\end{array}\right)
\end{aligned}
$$

and $\beta \in\{0,1\} . F_{j, k}^{V}$ is the convective volume flux density, $q_{j}$ is the value of $q \in\{J, \boldsymbol{\sigma}, \boldsymbol{\alpha}\}$ in the control volume (i.e. triangle) $\mathcal{V}_{j}$, and $q_{k}$ refers to the control volume $\mathcal{V}_{k}$ contiguous to the control volume $\mathcal{V}_{j}$ sharing the edge $\Gamma_{j, k} \stackrel{\text { def }}{=} \mathcal{V}_{j} \cap \mathcal{V}_{k}$. An edge is defined by the nodes $I$ and $I+1 \in\{0,1,2\}$, and node 2 is assumed to coincide with node 0 . Accordingly, $A_{j}$ is the area of $\mathcal{V}_{j}, R_{j}$ is the radial coordinate of the area centroid, $L_{j, k}$ is the length of edge $\Gamma_{j, k}, R_{j, k}$ is the radial coordinate of its centroid, $n_{1}, n_{2}$ are the components of the unit outward normal to an edge, and $c_{I}^{1}, c_{I}^{2}$ are the components of the 
convective velocity $\boldsymbol{c}$ at node $I$. A full donor-cell, first-order accurate upwind transport algorithm [38] is obtained when $\theta=1$.

\section{Numerical examples}

\subsection{Comparison of mesh smoothing algorithms}

The first example application of our ALE method should highlight the advantage of the proposed optimization-based mesh smoothing algorithm over heuristic algorithms extensively employed in the context of ALE methods. Heuristic (or direct) smoothing algorithms are not governed by quality evolution but provide closed-form expressions for the new coordinates of the nodes supposed to smooth the mesh, or a part of it. These methods usually fail if a mesh becomes non-convexly distorted, as this is the case in finite element simulations of penetration problems.

For reasons of comparison, two heuristic algorithms have been implemented which may replace the local algorithm for internal nodes formalized by (20). The first is based on weighted averaging and is applied in the ALE method of Aymone et al. [27]. The second algorithm has been developed by Giuliani [31] and is based on the analytical expression of the minimizer of a function describing the mesh distortion. For the example shown here, the results of both heuristic algorithms are comparable.

In order to create heavily distorted non-convex mesh regions, we take the simple example of backward extrusion of a compressible, hypoelastic material (Fig. 1). A billet is loaded into a container with rigid and smooth walls and then is moved towards a fixed rough die, so that the material is pushed through the die. By this, it suffices to discretize the billet only and to represent the die and the container walls using fixed and roller boundary conditions, respectively. The material behavior is described by a hypoelastic relation with constant isotropic elasticity tensor [43, eq. (5.6)].

Fig. 1 above shows the initial configuration together with the deformed mesh at $50 \%$ height reduction. The heavy squeezing of elements around the corner of the die cannot be avoided when using the heuristic method. The area of one element even vanishes, which inhibits convergence of the solution at continued extrusion. Compared to this, optimization-based smoothing achieves an excellent mesh regularization. At $50 \%$ height reduction, element squeezing is moderate, even in the non-convexly distorted region at the corner of the die. However, at continued extrusion the fixed mesh connectivity limits gains of mesh quality. Calculation terminates at height reductions of more 
heuristic smoothing

(Giuliani's method)

optimization-based

smoothing

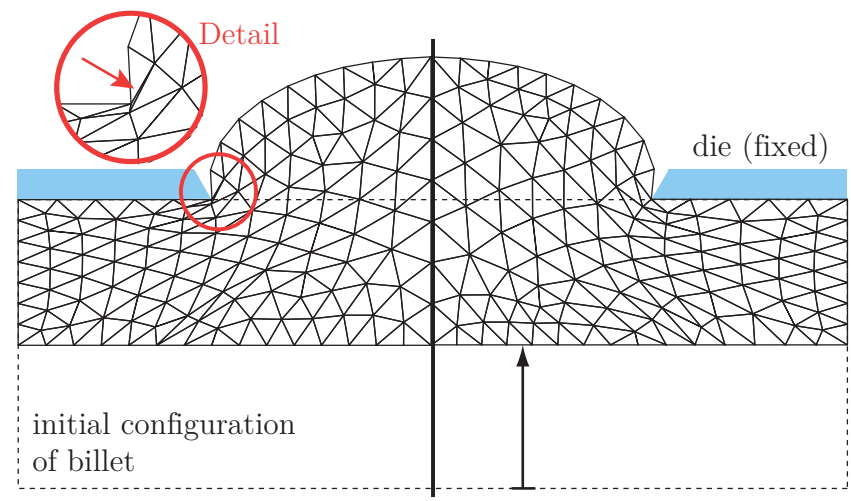

$50 \%$ reduction

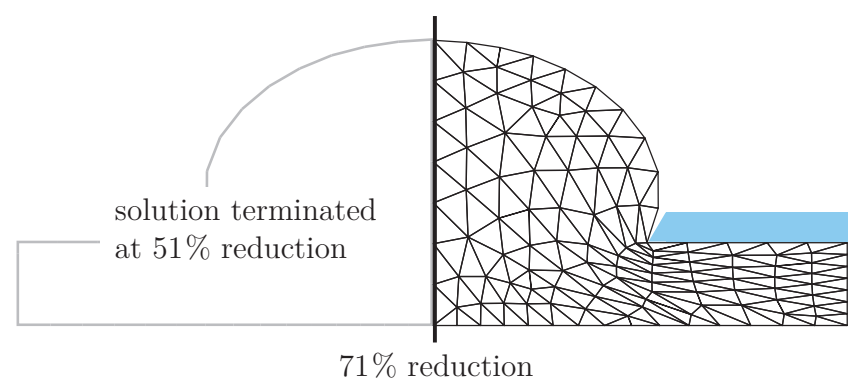

Figure 1: Comparison of an heuristic smoothing method and the developed optimizationbased algorithm when applied to a non-convexly distorted mesh. 
than $71 \%$. Only a complete remeshing would eliminate degenerate elements so as to continue solution.

\subsection{Shallow penetration into sand}

We now examine the capabilities of the proposed ALE method when applied to penetration into sand as a real world problem. In the two examples to be presented, the skin of the penetrator and the ground surface are modeled as a rigid-to-flexible contact pair using straight segments for the slave surface (ground surface) and accounting for large deformation of the interface. A Lagrange multiplier contact algorithm enforces zero penetration when contact is closed. As mentioned in the introductory section, the current version of the ALE method provides only smooth or perfectly rough contact. Surface roughness lying between these two extremes should be implemented into later versions due to its practical importance.

The first example is concerned with penetration of a strip footing into sand. Figure $2 \mathrm{a}$ shows the finite element model that has been designed with the intention to back-calculate the results of an experimental model test carried out at the Chair of Soil Mechanics and Geotechnical Engineering, TU Berlin; see [18] for details. The test was conducted in a chamber with observing window to digitally photograph the penetration process. Afterwards the captured image sequence was analyzed by particle image velocimetry (PIV) to reveal the soil motion without on-sample instrumentation.

The computational model assumes plain strain conditions and takes advantage of symmetry. The strip footing is rigid and perfectly rough, except for its sides which are assumed perfectly smooth. The initial relative density of the sand is $D_{\mathrm{r} 0}=78 \%$ (initial void ratio $e_{0}=0.546$ ). The hypoplastic rate constitutive equation is employed to model the behavior of the test sand; the material constants determined for the test sand are given in Appendix A. The assumed initial stress state within the sand is the $K_{0}$-state. It is calculated in an isotropically elastic dummy load step based on the bulk mass density and using a Poisson's ratio of $\nu=1 / 3\left(K_{0}=0.5\right)$.

For a relative penetration of $z / B=0.31$, where $B$ is the breadth of the foundation, the deformed mesh after smoothing is depicted in Fig. 2b. A direct comparison with Fig. 2a reveals that the node density at the corner of the strip footing is considerably increased during penetration. However, the uniform mesh retains a good quality because of the optimization-based mesh smoothing procedure. In contrast to that, running the ALE method in the purely Lagrangian mode causes the calculation to terminate just after a few 


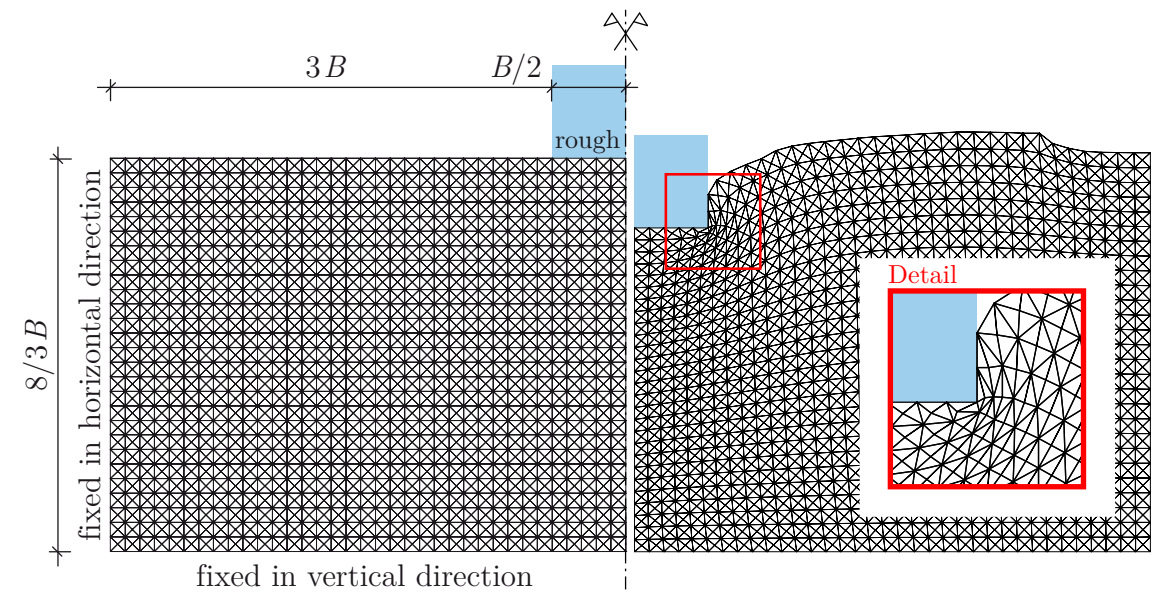

(a)

(b)

Figure 2: Shallow penetration into sand. (a) Problem statement and mesh, (b) deformed and smoothed mesh at a relative penetration depth of $z / B=0.31$.

time steps. We also tested other smoothing algorithms for internal meshes relying on heuristic approaches [27, 31], but only our new optimization-based algorithm led to a convergent solution for this example. The other two algorithms could not prevent elements to invert along with penetration, particularly at the indented mesh corner [28].

The advanced hypoplastic rate constitutive equation provides valuable insight into the mechanical behavior of sand. Consider, for example, the simulated void ratio distribution at $z / B=0.31$ plotted in Fig. 3. The results indicate that sand locally undergoes severe density changes during penetration. The initially dense sand loosens due to shearing lateral to the strip footing and along the slip surfaces; see also Fig. 4b. At the foundation corner, the reached void ratio of $e=0.81$ even lies above the maximum void ratio of $e_{\max }=0.779$ determined through laboratory tests. In the wedgeshaped zone underneath the foundation, on the other hand, the sand densifies along with penetration and reaches its minimum void ratio of $e_{\min }=0.482$.

The results of the ALE simulation are in good agreement with the backcalculated experiment, as can be seen from the comparative view in Fig. 4. The incremental displacement and the zones of maximum shear strain measured within the sand can be fairly reproduced. The measured (gray line) and the predicted ground heaving also match very well. Differences are found, however, in the shape of wedge underneath the pile base. The angle at the 


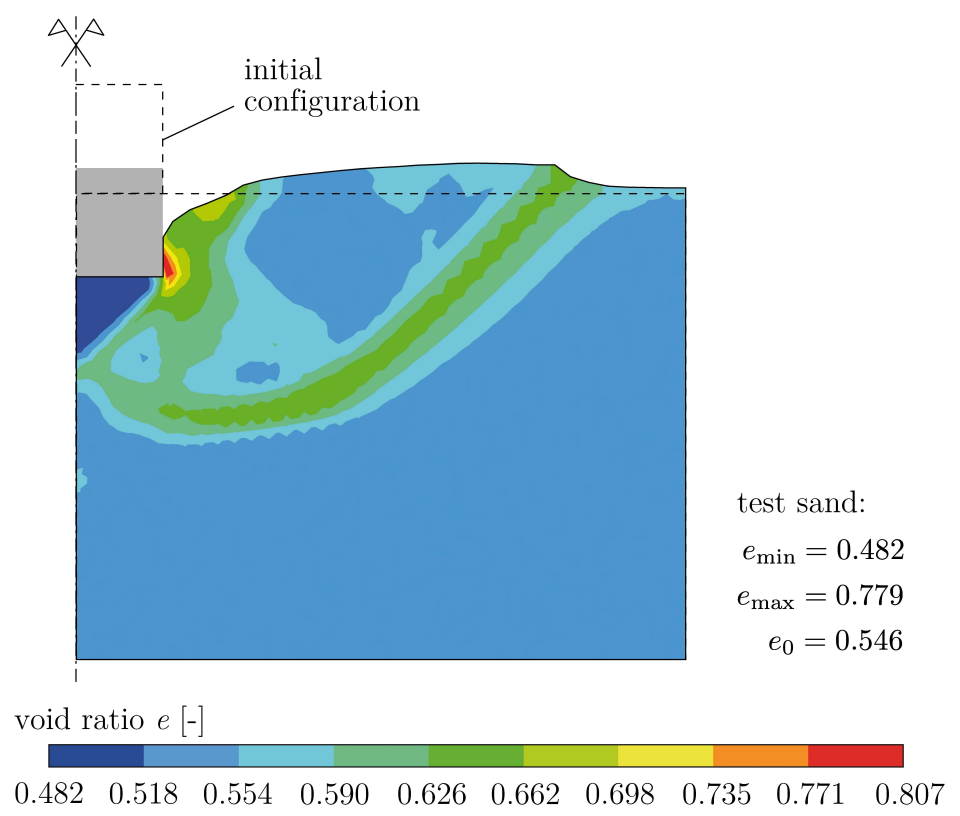

Figure 3: Shallow penetration into sand. Predicted distribution of the void ratio at a relative penetration depth of $z / B=0.31$.

apex of this zone is exactly $90^{\circ}$ in the simulation, whereas the experimental investigations indicate much larger angles.

While the experimental and numerical results are in good agreement with regard to incremental shear strain and ground heaving, the measured and predicted load-displacement curves plotted in Fig. 5 differ considerably when using the same set of hypoplastic material constants; $F_{\max }$ is the maximum penetration force of each load-displacement curve and is used for normalization. If we change the constant representing a reference pressure from $h_{\mathrm{s}}=76.5 \mathrm{MPa}$ to $765.0 \mathrm{MPa}$ (Appendix A) the load-displacement curves agree, but then ground heaving is overestimated. We have found that this undesirable feature is closely related to the capability of the constitutive equation to model dilatancy and the behavior of sand at very low levels of mean effective stress. In fact, under such conditions the shear resistance of any granular material is close to zero.

\subsection{Pile penetration into sand}

The final example deals with pile penetration and employs a zipper-type modeling technique. We chose a pile tip geometry which generally entails 


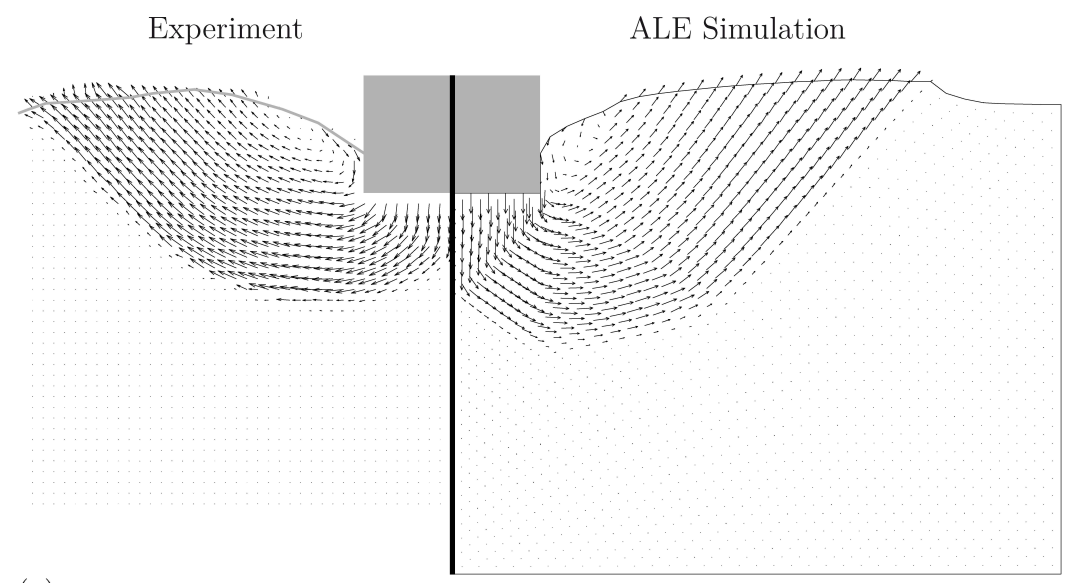

(a)

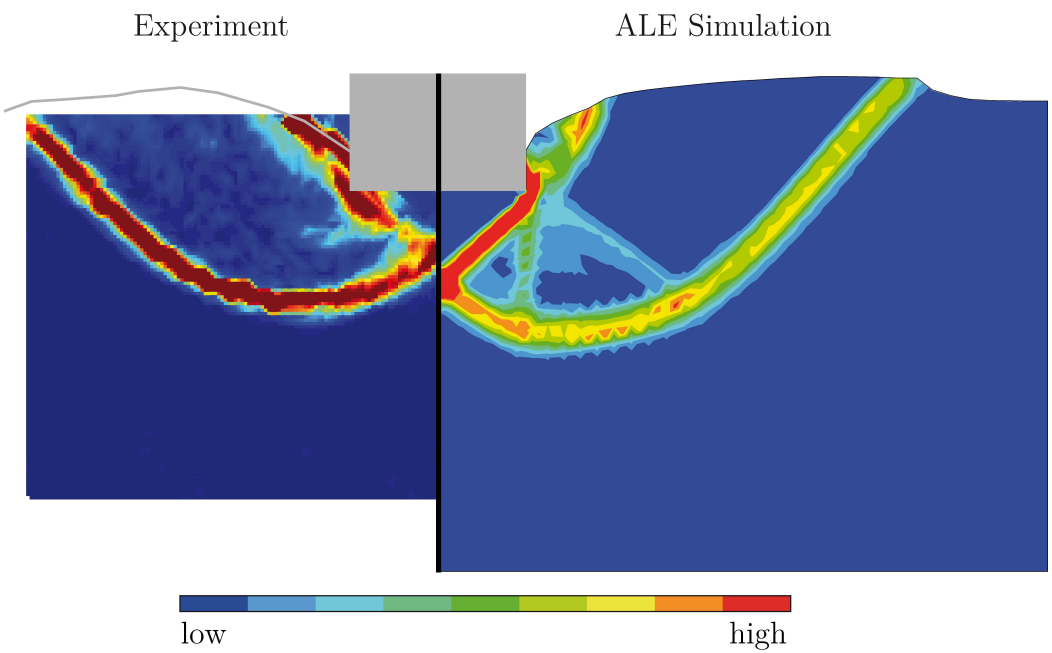

(b)

Figure 4: Comparison of the results of experimental test (PIV) and of the ALE backcalculation at $z / B=0.31$. (a) Incremental displacement, (b) incremental maximum shear strain. 


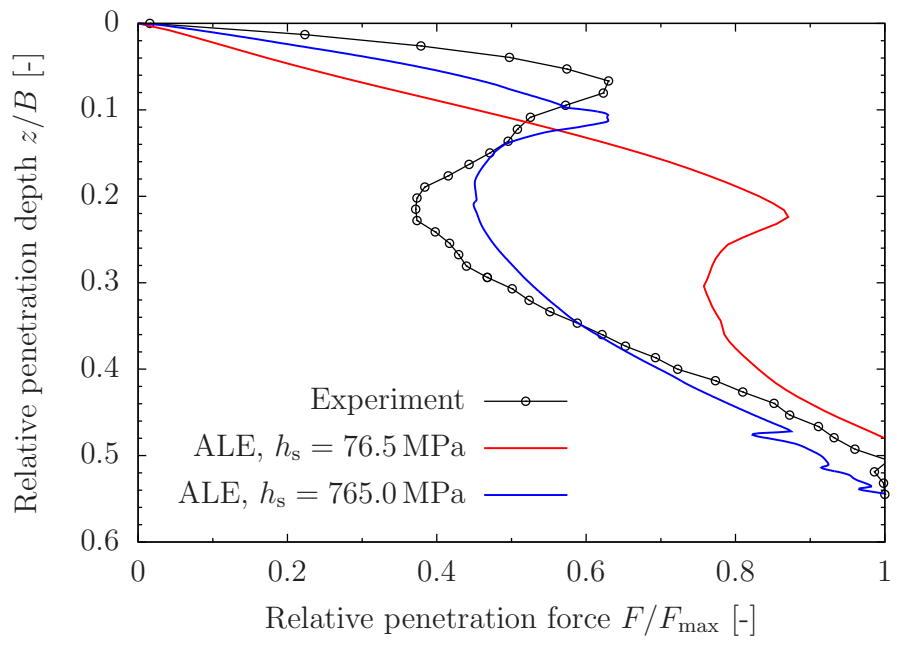

Figure 5: Comparison of the measured and predicted load-displacement curves of shallow penetration. $F_{\max }$ is the maximum penetration force of each load-displacement curve and is used for normalization, and $h_{\mathrm{s}}$ is a hypoplastic material constant representing a reference pressure (cf. Appendix A).

a great burden for Lagrangian methods and even for ALE methods. In the axisymmetric models set up (Fig. 6), the "pile" is designed as a segment of a rigid slideline supporting the soil in the direction normal to the slideline. Smooth contact conditions are assumed between the soil and the pile to approximate the surface roughness of steel or precast concrete piles. One unstructured and two structured finite element meshes were generated (Fig. 6). A $K_{0}=0.5$ initial stress state within the sand is assumed in accordance with the previous example, and the initial relative density of the sand is $D_{\mathrm{r} 0}=34 \%$ (initial void ratio $e_{0}=0.678$ ).

Numerical results of the succeeded ALE simulations using the structured fine mesh are plotted in Fig. 7. The sand significantly densifies underneath the pile base at all stages of penetration. The pile shaft is surrounded by a zone of severe loosening during the course of penetration. The zone of maximum loosening is located between $0.5 D$ and $1.0 D$ below the ground surface irrespective of the penetration depth. Within this zone, $e \approx 0.81$ which corresponds to a negative relative density (cf. Appendix A). However, in the major part of the soil domain the sand is compressed such that the volume of the ground heaving is outbalanced by the volume of the penetrated pile. 


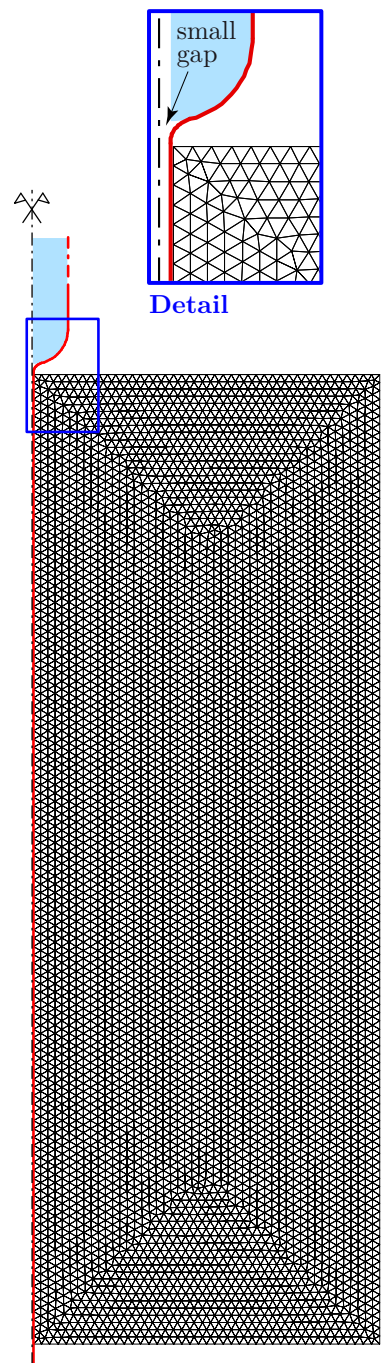

(a) 26000 DOF
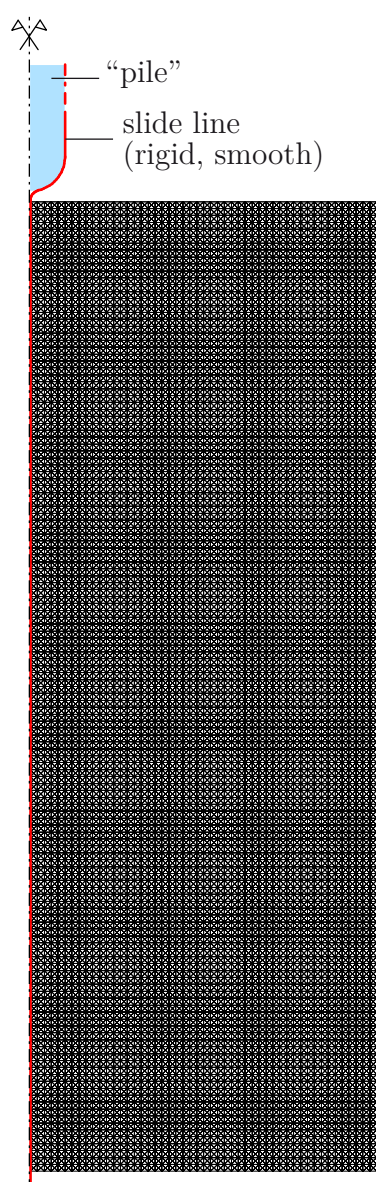

(b) 30000 DOF

Figure 6: Pile penetration into sand. Problem statement and (a) initial unstructured coarse mesh, (b) initial structured coarse mesh. The structured fine mesh with $\sim 65000$ DOF is not shown. 

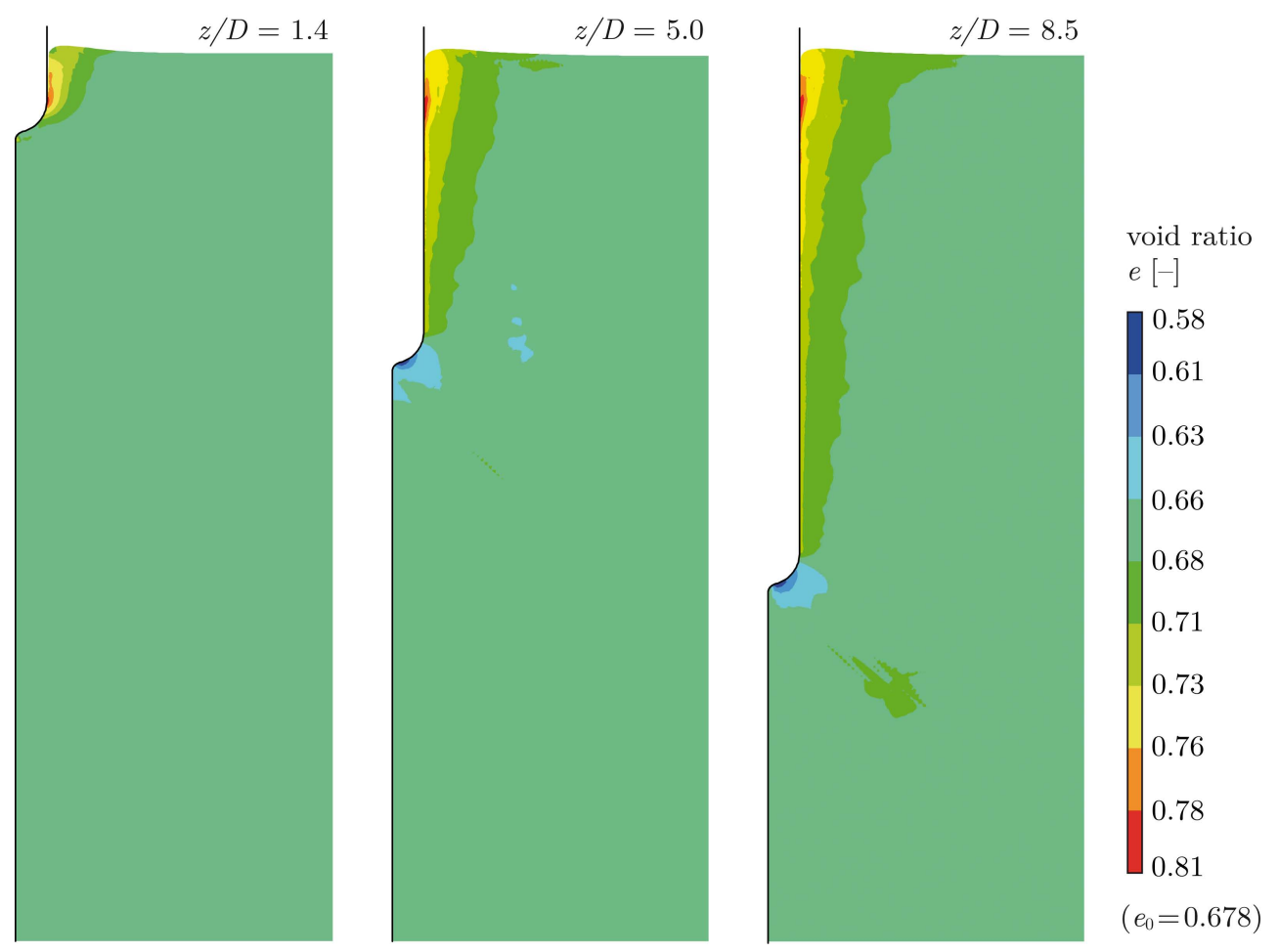

Figure 7: Pile penetration into sand. Void ratio distributions at different penetration depths. 


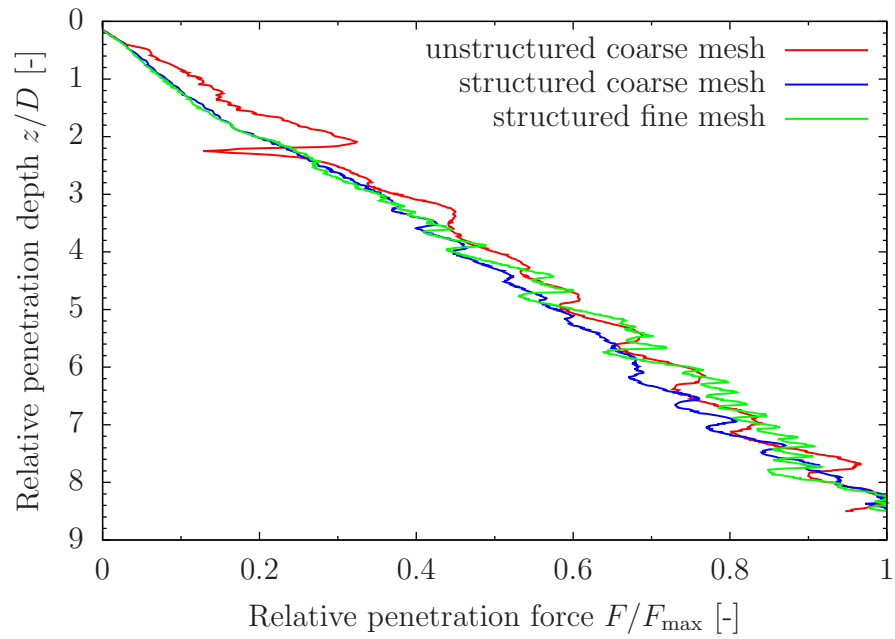

Figure 8: Relative load-displacement curves of simulated pile penetration. $F_{\max }$ is the maximum penetration force of each load-displacement curve and is used for normalization.

The relative load-displacement curves using the maximum penetration force $F_{\max }$ of each curve as a normalization factor are plotted in Fig. 8. At penetration depths larger than three times the pile diameter, the curves are relatively linear and show a characteristic zig-zag form which is a manifestation of spurious oscillation. This unrealistic behavior is governed by the quality of geometry representation at the interfaces in the finite element model. More clearly, the pile skin (master) is modeled as a smooth curve while the boundary of the soil domain (slave) is covered with straight segments. During penetration some nodes of the slave surface lose contact with the pile skin when the pile tip passes, causing a periodical release of the finite element nodal forces. It can be seen from the figure that the poorest discretization associated with the unstructured coarse mesh tends to the highest oscillation.

\section{Conclusions}

A comprehensive arbitrary Lagrangian-Eulerian framework for the numerical simulation of large deformation problems with special consideration of plane strain and axisymmetric quasi-static penetration into sand has been presented. The implicit ALE finite element method has been combined with an advanced hypoplastic rate constitutive equation which predicts the me- 
chanical behavior of sand quite realistically. Such complex constitutive equations are unusual in the traditional application areas of ALE. Moreover, an efficient optimization technique has been implemented which smoothes out the non-convexly distorted mesh regions that occur along with penetration.

The assumptions and restrictions associated with the present work listed in Sect. 1 give motivation to further research. For example, the incorporation of inertia effects in the developed ALE method is of great practical importance because displacement piles are usually driven by hammering or vibration. Further improvement of the presented ALE method would be the provision for surface roughness in the contact model at the soil-penetrator interface. This would introduce contact history variables that must be properly remapped onto the smoothed mesh during the transport step of the method.

Another relevant aspect of research in the area of soil mechanical ALE methods is the interaction of the solid phase with one or more pore fluids. Contrary to the assumptions inherent to the current method, sand in situ is rarely dry or fully saturated and locally drained. Therefore, many important phenomena cannot be reproduced. Taking into account the soil as a two-phase or even three-phase medium would enable a large deformation analysis of problems involving liquefaction-prone and/or partially saturated soils under cyclic loading, for example slopes and dams under earthquake excitation and driving of piles for offshore foundation systems.

\section{Acknowledgements}

The presented research work was carried out under the financial support from the German Research Foundation (DFG), grants SA 310/21-1 and SA 310/21-2, which is gratefully acknowledged.

\section{Appendix A. Hypoplastic model for isotropic sand}

This paper considers only effective stress, which is equal to the total stress in the absence of pore pressure (dry conditions). The common sign convention of general mechanics is applied, i.e. compressive stress or strain is taken with negative sign, and pressure has positive sign whenever stress is compressive.

In our ALE method we use a version of the hypoplastic rate constitutive

equation proposed by Niemunis and Herle [25]. Its mathematical formulation 
can be summarized as

$$
\begin{aligned}
\stackrel{\nabla}{\boldsymbol{\sigma}} & =\boldsymbol{m}(\boldsymbol{\sigma}, e, \boldsymbol{\delta}, \boldsymbol{d}): \boldsymbol{d} \\
\stackrel{\nabla}{\boldsymbol{\delta}} & =\boldsymbol{j}(\boldsymbol{\delta}, \boldsymbol{d}) \\
\dot{e} & =(1+e) \operatorname{tr} \boldsymbol{d}
\end{aligned}
$$

where the second-order tensor $\boldsymbol{\delta}(x, t)$ is the so-called intergranular strain and $e(x, t)$ is the spatial void ratio, which is the ratio of the partial volume of interconnected voids and the partial solid volume in a representative volume element, and $\stackrel{\nabla}{s}$ is the Zaremba-Jaumann rate of $\boldsymbol{s}$. The intergranular strain is an additional internal state variable representing a macroscopic measure of micro-deformations of the interface zone between sand grains. Moreover,

$$
\begin{aligned}
& \boldsymbol{m}(\boldsymbol{\sigma}, e, \boldsymbol{\delta}, \boldsymbol{d}) \stackrel{\text { def }}{=}\left(\rho^{\chi} m_{\mathrm{T}}+\left(1-\rho^{\chi}\right) m_{\mathrm{R}}\right) \boldsymbol{L} \\
& + \begin{cases}\rho^{\chi}\left(1-m_{\mathrm{T}}\right) \boldsymbol{L}: \overrightarrow{\boldsymbol{\delta}} \otimes \overrightarrow{\boldsymbol{\delta}}+\rho^{\chi} \boldsymbol{N} \otimes \overrightarrow{\boldsymbol{\delta}} & \text { for } \overrightarrow{\boldsymbol{\delta}}: \boldsymbol{d}>0 \\
\rho^{\chi}\left(m_{\mathrm{R}}-m_{\mathrm{T}}\right) \boldsymbol{L}: \overrightarrow{\boldsymbol{\delta}} \otimes \overrightarrow{\boldsymbol{\delta}} & \text { for } \overrightarrow{\boldsymbol{\delta}}: \boldsymbol{d} \leq 0,\end{cases} \\
& \boldsymbol{j}(\boldsymbol{\delta}, \boldsymbol{d}) \stackrel{\text { def }}{=} \begin{cases}\boldsymbol{d}-\rho^{\beta_{\mathrm{r}}} \overrightarrow{\boldsymbol{\delta}} \otimes \overrightarrow{\boldsymbol{\delta}}: \boldsymbol{d} & \text { for } \overrightarrow{\boldsymbol{\delta}}: \boldsymbol{d}>0 \\
\boldsymbol{d} & \text { for } \overrightarrow{\boldsymbol{\delta}}: \boldsymbol{d} \leq 0\end{cases} \\
& \boldsymbol{L}(\tilde{\boldsymbol{\sigma}}) \stackrel{\text { def }}{=} \frac{f_{\mathrm{b}} f_{\mathrm{e}}}{\operatorname{tr}\left(\tilde{\boldsymbol{\sigma}}^{2}\right)}\left(F^{2} \mathbf{1}+a^{2} \tilde{\boldsymbol{\sigma}} \otimes \tilde{\boldsymbol{\sigma}}\right), \\
& \boldsymbol{N}(\tilde{\boldsymbol{\sigma}}) \stackrel{\text { def }}{=} f_{\mathrm{b}} f_{\mathrm{e}} f_{\mathrm{d}} \frac{a F}{\operatorname{tr}\left(\tilde{\boldsymbol{\sigma}}^{2}\right)}\left(\tilde{\boldsymbol{\sigma}}+\tilde{\boldsymbol{\sigma}}_{\mathrm{dev}}\right) \text {, } \\
& \mathbf{1} \stackrel{\text { def }}{=} \frac{1}{2}(\boldsymbol{I} \otimes \boldsymbol{I}+\boldsymbol{I} \otimes \boldsymbol{I}), \\
& F \stackrel{\text { def }}{=} \sqrt{\frac{1}{8} \tan ^{2} \psi+\frac{2-\tan ^{2} \psi}{2+\sqrt{2} \tan \psi \cos 3 \theta}}-\frac{1}{2 \sqrt{2}} \tan \psi \\
& a \stackrel{\text { def }}{=} \frac{\sqrt{3}\left(3-\sin \phi_{\mathrm{c}}\right)}{2 \sqrt{2} \sin \phi_{\mathrm{c}}} \\
& \rho \stackrel{\text { def }}{=} \frac{\|\boldsymbol{\delta}\|}{R}, \quad \tan \psi \stackrel{\text { def }}{=} \sqrt{3}\left\|\tilde{\boldsymbol{\sigma}}_{\text {dev }}\right\|, \quad \cos 3 \theta \stackrel{\text { def }}{=}-\sqrt{6} \frac{\operatorname{tr}\left(\tilde{\boldsymbol{\sigma}}_{\text {dev }}^{3}\right)}{\left(\operatorname{tr}\left(\tilde{\boldsymbol{\sigma}}_{\text {dev }}^{2}\right)\right)^{\frac{3}{2}}},
\end{aligned}
$$




$$
\begin{gathered}
\tilde{\boldsymbol{\sigma}} \stackrel{\text { def }}{=}-\frac{\boldsymbol{\sigma}}{3 p}, \quad p \stackrel{\text { def }}{=}-\frac{1}{3} \operatorname{tr} \boldsymbol{\sigma}, \quad \tilde{\boldsymbol{\sigma}}_{\mathrm{dev}} \stackrel{\text { def }}{=} \tilde{\boldsymbol{\sigma}}-\frac{1}{3} \boldsymbol{I}, \quad \overrightarrow{\boldsymbol{\delta}} \stackrel{\text { def }}{=} \frac{\boldsymbol{\delta}}{\|\boldsymbol{\delta}\|}, \\
f_{\mathrm{e}}(\boldsymbol{\sigma}, e) \stackrel{\text { def }}{=}\left(\frac{e_{\mathrm{c}}}{e}\right)^{\beta}, \\
f_{\mathrm{d}}(\boldsymbol{\sigma}, e) \stackrel{\text { def }}{=}\left(\frac{e-e_{\mathrm{d}}}{e_{\mathrm{c}}-e_{\mathrm{d}}}\right)^{\alpha}, \quad \text { and } \\
f_{\mathrm{b}}(\boldsymbol{\sigma}) \stackrel{\text { def }}{=} \frac{h_{\mathrm{s}}}{n}\left(\frac{1+e_{\mathrm{i}}}{e_{\mathrm{i}}}\right)\left(\frac{e_{\mathrm{i} 0}}{e_{\mathrm{c} 0}}\right)^{\beta}\left(\frac{3 p}{h_{\mathrm{s}}}\right)^{1-n}\left(3+a^{2}-\sqrt{3} a\left(\frac{e_{\mathrm{i} 0}-e_{\mathrm{d} 0}}{e_{\mathrm{c} 0}-e_{\mathrm{d} 0}}\right)^{\alpha}\right)^{-1},
\end{gathered}
$$

with the second-order unit tensor $\boldsymbol{I}$ and the characteristic void ratios $e_{\mathrm{i}}(p)$, $e_{\mathrm{c}}(p)$, and $e_{\mathrm{d}}(p)$ being obtained through Bauer's formula [23]

$$
\frac{e_{\mathrm{i}}}{e_{\mathrm{i} 0}} \stackrel{\text { def }}{=} \frac{e_{\mathrm{c}}}{e_{\mathrm{c} 0}} \stackrel{\text { def }}{=} \frac{e_{\mathrm{d}}}{e_{\mathrm{d} 0}} \stackrel{\text { def }}{=} \exp \left(-\left(\frac{3 p}{h_{\mathrm{s}}}\right)^{n}\right) \text {. }
$$

The hypoplastic model contains 13 material constants: $\phi_{\mathrm{c}}$ is the friction angle at critical state (in $\left.{ }^{\circ}\right), h_{\mathrm{s}}$ is a reference pressure called granulate hardness (in $\mathrm{Pa}), e_{\mathrm{d} 0}$ and $e_{\mathrm{i} 0}$ are the minimum and maximum void ratio at zero pressure, respectively, $e_{\mathrm{c} 0}$ is the void ratio at critical state at zero pressure, $R$ is the maximum magnitude of intergranular strain, $m_{\mathrm{R}}, m_{\mathrm{T}}$ are stiffness factors associated with loading reversals, and $n, \alpha, \beta, \beta_{\mathrm{r}}, \chi$ are exponents. The determination of these material constants from experimental laboratory test is roughly described in the articles cited. More detailed information can be found in $[26,44]$.

For the back-analysis of the experimental model test presented in Section 5, a complete set of hypoplastic material constant for that sand used in the tests had to be determined. These constants are: $\phi_{\mathrm{c}}=31.5^{\circ}, h_{\mathrm{s}}=$ $76500 \mathrm{MPa}\left(h_{\mathrm{s}}=76.5 \mathrm{MPa}\right.$ for $\left.0 \mathrm{kPa}<p<20 \mathrm{kPa}\right), n=0.29, e_{\mathrm{d} 0}=0.48$, $e_{\mathrm{c} 0}=0.78, e_{\mathrm{i} 0}=0.90, \alpha=0.13, \beta=1.0, R=1 \times 10^{-4}, m_{\mathrm{R}}=5.0, m_{\mathrm{T}}=2.0$, $\beta_{\mathrm{r}}=0.5, \chi=6.0$. Some of these values were estimated in accordance with the references cited.

\section{References}

[1] J. G. Trulio, Theory and structure of the AFTON codes, Report AFWLTR-66-19, Air Force Weapons Laboratory, Kirtland Air Force Base, New Mexico, USA (1966). 
[2] C. W. Hirt, A. A. Amsden, J. L. Cook, An arbitrary LagrangianEulerian computing method for all flow speeds, Journal of Computational Physics 14 (1974) 227-253.

[3] T. J. R. Hughes, W. K. Liu, T. K. Zimmermann, Lagrangian-Eulerian finite element formulation for incompressible viscous flows, Computer Methods in Applied Mechanics and Engineering 29 (1981) 329-349.

[4] W. K. Liu, T. Belytschko, H. Chang, An arbitrary Lagrangian-Eulerian finite element method for path-dependent materials, Computer Methods in Applied Mechanics and Engineering 58 (1986) 227-245.

[5] D. J. Benson, An efficient, accurate, simple ALE method for nonlinear finite element programs, Computer Methods in Applied Mechanics and Engineering 72 (3) (1989) 305-350.

[6] A. Rodríguez-Ferran, F. Casadei, A. Huerta, ALE stress update for transient and quasistatic processes, International Journal for Numerical Methods in Engineering 43 (1998) 241-262.

[7] E. Susila, R. D. Hryciw, Large displacement FEM modelling of the cone penetration test (CPT) in normally consolidated sand, International Journal for Numerical and Analytical Methods in Geomechanics 27 (2003) 585-602.

[8] D. S. Liyanapathirana, Arbitrary Lagrangian Eulerian based finite element analysis of cone penetration in soft clay, Computers and Geotechnics 36 (2009) 851-860.

[9] M. Nazem, D. Sheng, J. P. Carter, Stress integration and mesh refinement for large deformation in geomechanics, International Journal for Numerical Methods in Engineering 65 (2006) 1002-1027.

[10] M. Nazem, D. Sheng, J. P. Carter, S. W. Sloan, Arbitrary LagrangianEulerian method for large-strain consolidation problems, International Journal for Numerical and Analytical Methods in Geomechanics 32 (9) (2008) 1023-1050.

[11] D. Sheng, M. Nazem, J. P. Carter, Some computational aspects for solving deep penetration problems in geomechanics, Computational Mechanics 44 (2009) 549-561. 
[12] Y. Di, J. Yang, T. Sato, An operator-split ALE model for large deformation analysis of geomaterials, International Journal for Numerical and Analytical Methods in Geomechanics 31 (2007) 1375-1399.

[13] O. C. Zienkiewicz, A. H. C. Chan, M. Pastor, B. A. Schrefler, T. Shiomi, Computational Geomechanics with Special Reference to Earthquake Engineering, John Wiley \& Sons, Ltd., 1999.

[14] X. S. Li, Y. F. Dafalias, Dilatancy for cohesionless soils, Géotechnique 50 (4) (2000) 449-460.

[15] M. Goldscheider, G. Gudehus, Rectilinear extension of dry sand: Testing apparatus and experimental results, in: Proceedings 8th International Conference on Soil Mechanics and Foundation Engineering, Moscow, Vol. 1, 1973, pp. 143-149.

[16] T. Belytschko, W. K. Liu, B. Moran, Nonlinear Finite Elements for Continua and Structures, John Wiley \& Sons, Ltd., 2000.

[17] P. Wriggers, Nonlinear Finite Element Methods, Springer-Verlag Berlin Heidelberg, 2008.

[18] D. Aubram, An Arbitrary Lagrangian-Eulerian Method for Penetration into Sand at Finite Deformation, no. 62 in Veröffentlichungen des Grundbauinstitutes der Technischen Universität Berlin, Shaker Verlag, Aachen, 2013, http://opus4.kobv.de/opus4-tuberlin/frontdoor/index/index/docId/4755.

[19] D. J. Benson, Computational methods in Lagrangian and Eulerian hydrocodes, Computer Methods in Applied Mechanics and Engineering 99 (2-3) (1992) 235-394.

[20] D. Kolymbas, An outline of hypoplasticity, Archive of Applied Mechanics 61 (3) (1991) 143-151.

[21] C. Truesdell, W. Noll, The Non-Linear Field Theories of Mechanics, 3rd Edition, Springer-Verlag Berlin Heidelberg New York, 2004.

[22] G. Gudehus, A comprehensive constitutive equation for granular materials, Soils and Foundations 36 (1) (1996) 1-12. 
[23] E. Bauer, Calibration of a comprehensive constitutive equation for granular materials, Soils and Foundations 36 (1) (1996) 13-26.

[24] P.-A. von Wolffersdorff, A hypoplastic relation for granular materials with a predefined limit state surface, Mechanics of Cohesive-Frictional Materials 1 (1996) 251-271.

[25] A. Niemunis, I. Herle, Hypoplastic model for cohesionless soils with elastic strain range, Mechanics of Cohesive-Frictional Materials 2 (1997) 279-299.

[26] I. Herle, G. Gudehus, Determination of parameters of a hypoplastic constitutive model from properties of grain assemblies, Mechanics of Cohesive-Frictional Materials 4 (1999) 461-486.

[27] J. L. F. Aymone, E. Bittencourt, G. J. Creus, Simulation of 3d metalforming using an arbitrary Lagrangian-Eulerian finite element method, Journal of Materials Processing Technology 110 (2001) 218-232.

[28] D. Aubram, Optimization-based smoothing algorithm for triangle meshes over arbitrarily shaped domains, arXiv:1410.5977 [cs.NA] (2014).

[29] T. J. R. Hughes, J. Winget, Finite rotation effects in numerical integration of rate constitutive equations arising in large-deformation analysis, International Journal for Numerical Methods in Engineering 15 (12) (1980) 1862-1867.

[30] T. J. R. Hughes, Numerical implementation of constitutive models: Rate-independent deviatoric plasticity, in: S. Nemat-Nasser, R. J. Asaro, G. A. Hegemier (Eds.), Theoretical Foundation for Large-Scale Computations for Nonlinear Material Behavior, Martinus Nijhoff Publishers, Dordrecht, Niederlande, 1984, pp. 29-63.

[31] S. Giuliani, An algorithm for continuous rezoning of the hydrodynamic grid in arbitrary Lagrangian-Eulerian computer codes, Nuclear Engineering and Design 72 (1982) 205-212.

[32] R. Löhner, K. Morgan, O. C. Zienkiewicz, Adaptive grid refinement for the compressible Euler equations, in: I. Babuška, O. C. Zienkiewicz, J. Gago, E. R. A. Oliviera (Eds.), Accuracy Estimates and Adaptive 
Refinements in Finite Element Computations, John Wiley \& Sons, Ltd., 1986, pp. 281-297.

[33] E. A. Dari, G. C. Buscaglia, Mesh optimization: How to obtain good unstructured 3d finite element meshes with not-so-good mesh generators, Structural and Multidisciplinary Optimization 8 (1993) 181-188.

[34] P. D. Zavattieri, E. A. Dari, G. C. Buscaglia, Optimization strategies in unstructured mesh generation, International Journal for Numerical Methods in Engineering 39 (1996) 2055-2071.

[35] H. Braess, P. Wriggers, Arbitrary Lagrangian Eulerian finite element analysis of free surface flow, Computer Methods in Applied Mechanics and Engineering 190 (2000) 95-109.

[36] P. M. Knupp, L. G. Margolin, M. Shashkov, Reference Jacobian optimization-based rezone strategies for arbitrary Lagrangian Eulerian methods, Journal of Computational Physics 176 (2002) 93-128.

[37] W. Sun, Y.-X. Yuan, Optimization Theory and Methods - Nonlinear Programming, Springer Science+Business Media, LLC, 2006.

[38] R. J. LeVeque, Finite Volume Methods for Hyperbolic Problems, 3rd Edition, Cambridge University Press, Cambridge, UK, 2002.

[39] E. F. Toro, Riemann Solvers and Numerical Methods for Fluid Dynamics - A Practical Introduction, 2nd Edition, Springer-Verlag Berlin Heidelberg, 1999.

[40] H. Askes, L. Bodé, L. J. Sluys, ALE analysis of localization in wave propagation problems, Mechanics of Cohesive-Frictional Materials 3 (1998) 105-125.

[41] D. Freßmann, P. Wriggers, Advection approaches for single- and multimaterial arbitrary Lagrangian-Eulerian finite element procedures, Computational Mechanics 39 (2007) 153-190.

[42] F. L. Addessio, J. R. Baumgardner, J. K. Dukowicz, N. L. Johnson, B. A. Kashiwa, R. M. Rauenzahn, C. Zemach, CAVEAT: A Computer Code for Fluid Dynamics Problems with Large Distortion and Internal Slip, Report LA-10613-MS-REV. 1, Los Alamos National Laboratory, Los Alamos, USA, (revised edition) (1990). 
[43] J. C. Simo, K. S. Pister, Remarks on rate constitutive equations for finite deformation problems: Computational implications, Computer Methods in Applied Mechanics and Engineering 46 (1984) 201-215.

[44] F. Rackwitz, Numerische Untersuchungen zum Tragverhalten von Zugpfählen und Zugpfahlgruppen in Sand auf der Grundlage von Probebelastungen, Dissertation, Fakultät VI - Bauingenieurwesen und Angewandte Geowissenschaften, Technische Universität Berlin, Germany, (published in Institute Series; in German) (2003). 